\title{
Comparison of wound healing effects between Oncorhynchus keta-derived polydeoxyribonucleotide (PDRN) and Oncorhynchus mykiss-derived PDRN
}

Jong Hun Lee $e^{1,2^{*}}$,
Jin Woo Han' ${ }^{1^{*}}$,
Jin Hwan Byun',
Won Mi Lee
Min Ho Kim²,
Wen Hao Wu

${ }^{1}$ Department of Plastic and Reconstructive Surgery, Eulji General Hospital, Eulji University School of Medicine, Seoul;

${ }^{2}$ Eulji Medi-Bio Research Institute, Eulji University, Seoul;

${ }^{3}$ Department of Pathology, Eulji General Hospital, Eulji University School of Medicine, Seoul, Korea

\begin{abstract}
Background: Polydeoxyribonucleotide (PDRN) influencing cellular growth and differentiation is recognized to promote wound healing by stimulating tissue repair. Although PDRN can be extracted from human placentas, PDRN medications have recently been extracted from the semen of trout (Oncorhynchus mykiss) and salmon (Oncorhynchus keta). The present study was designed to evaluate the wound healing effects of $O$. keta-derived PDRN for injection (Rejuvenex) and PDRN cream (Rejuvenex Cream) in comparison with those of $O$. mykiss-derived PDRN injection (Placentex).

Methods: Full-thickness skin defects were made on the back of mice $(n=60)$. The mice were divided into the following four groups according to the dressing used for the wounds: O. mykiss-derived PDRN injection group, O. keta-derived PDRN injection group, O. ketaderived PDRN cream group, and normal saline soaked dressing group (control group). We analyzed the gross findings, wound sizes, histological findings, immunohistochemistry and enzyme-linked immunosorbent assays for the groups immediately after the treatment, and again after 4,7 , and 10 days of treatment.

Results: The wound healing effects were the greatest in the $O$. keta-derived PDRN injection and $O$. mykiss-derived PDRN injection groups, which showed similar scores, followed by the $O$. keta-derived cream and normal saline soaked dressing groups.

Conclusion: The injection of PDRN extracted from $O$. keta was found to be as effective at healing full-thickness skin defects as the $O$. mykiss-derived PDRN injection, which is currently used in the clinic. Moreover, the $O$. keta-derived PDRN injection was also found to reduce the time required for wound healing.
\end{abstract}

Keywords: Polydeoxyribonucleotide / Salmon / Defect / Wound healing

\footnotetext{
Correspondence: Jong Hun Lee

Department of Plastic and Reconstructive Surgery, Eulji General Hospital, Eulji University

School of Medicine, 68 Hangeulbiseong-ro, Nowon-gu, Seoul 01830, Korea

E-mail: joaljh@eulji.ac.kr

*The first two authors contributed equally to this work.

*This work was supported by Eulji University Industry-Academia Collaboration

Foundation Grants (No. 13-023-411) from the Eulji University.
}

Author contribution: Research conception \& design: JH Lee; performing the experiments: JH Lee, MH Kim, WW Hao; data acquisition: JH Lee, JW Han, JH Byun, WM Lee; data analysis and interpretation: JH Lee, JW Han, JH Byun, WM Lee, MH Kim, WW Hao; statistical analysis: JW Han; drafting of the manuscript: JH Lee, JW Han; critical revision of the manuscript: JH Lee, JW Han; receiving grant: JH Lee; approval of final manuscript: all authors.

Received January 30, 2018 / Revised March 14, 2018 / Accepted March 15, 2018

\section{INTRODUCTION}

Wound healing occurs through multiple stages of coagulation, inflammation, proliferation, and remodeling, which are accompanied by re-epithelialization, contraction, the formation of granulation tissue, and collagen synthesis [1-4]. Currently, various dressings and treatment methods utilizing diverse substances have been developed in an attempt to treat excisional wounds of the skin. 
Human placenta-derived natural substances, such as hormones and glycosaminoglycan, are used to treat various diseases and conditions. Many biochemicals have been developed as synthetic drugs. Of interest, studies have reported that DNA fragments extracted from human placentas can play positive roles in various diseases, with particular benefits for tissue regeneration. Subsequent studies identified polydeoxyribonucleotide (PDRN), which regulates cytokines and growth factors, and novel extraction methods have been devised to extract PDRN from natural substances. PDRN contains deoxyribonucleotide polymers and is a DNA fragment of 50-2,000 bases. It initiates intracellular signaling through A2 purinergic receptors, which are a type of Gprotein coupled receptors. Numerous studies have shown that PDRN also serves as an energy source, thus influencing cellular growth and differentiation. The activation of A2 receptors is known to be associated with tissue regeneration and anti-inflammatory effects, and is recognized to promote wound healing by stimulating tissue repair. Although PDRN can be extracted from human placentas, PDRN medications have recently been extracted from the semen of trout (Oncorhynchus mykiss) and salmon (Oncorhynchus keta) [5,6]. Such medications are obtained through high-temperature extraction and are characterized by a purity $>95 \%$ without any additional pharmacologically-active proteins or peptides (Registration Dossier, Italian Ministry of Health) [7]. In terms of the chemical structure, PDRN is composed of low-molecular weight DNA and is a linear polymer comprising 50-2,000 base pairs. PDRN is degraded by active membrane enzymes to serve as a source of purine and pyrimidine deoxynucleosides and deoxyribonucleotides, which increase cellular proliferation and activity in different tissues [8-12].

A PDRN injection can be used in the clinical setting to treat wounds, including diabetic foot ulcers. This treatment is already being prepared from the semen of $O$. mykiss, and is imported from Italy (Placentex Integro, Mastelli, Sanremo, Italy). In South Korea, PDRN has been extracted and purified from the semen of chum salmon (O. keta), which belongs to the same species as $O$. mykiss and returns to South Korea for spawning; the equivalence of PDRN from difference sources has been confirmed [9,13]. Further, PDRN for injection (Rejuvenex, Pharma-Research, Seong- nam, Korea) and a PDRN cream (Rejuvenex Cream, Pharma-Research) have been developed from the semen of $O$. keta.

In order to provide basic data for the development of new $O$. keta-derived PDRN products for wound healing, the present study was designed to evaluate the wound healing effects of $O$. keta-derived PDRN for injection (Rejuvenex) and PDRN cream (Rejuvenex Cream) in comparison with those of $O$. mykiss-derived PDRN injection (Placentex). For this purpose, full-thickness skin defects were created in white mice, and the effects of different PDRN-based treatments were evaluated.

\section{METHODS}

\section{Animal models}

The experiments were conducted in accordance with the regulations of the Animal Care and Use Committee of Eulji University (No. 13-15) that oversees animal experiments. Sixty-five of 6-weekold male white ICR (Crl:CD1) mice were used for the experiments. All mice were kept in a laboratory room with optimized environmental conditions (temperature, $24^{\circ} \mathrm{C} \pm 2^{\circ} \mathrm{C}$; humidity, $50 \% \pm 10 \%$ ) under a $12 \mathrm{hr} /$ day light cycle. Food and water were available ad libitum. Prior to conducting experiments, the mice were allowed to acclimatize to the environment for at least 1 week.

\section{Materials}

Materials are as follows: (1) O. mykiss-derived PDRN injection: 1.875 w/v\% PDRN solution (Placentex); (2) O. keta-derived PDRN injection: $1.875 \mathrm{w} / \mathrm{v} \%$ PDRN solution (Rejuvenex, Pharma-Research); (3) O. keta-derived PDRN cream: 0.08 w/w\% PDRN cream (Rejuvenex Cream, Pharma-Research); (4) normal saline soaking: $0.9 \% \mathrm{NaCl}$ solution.

\section{Methods}

\section{Full-thickness skin defects and PDRN procedures}

Sixty-five ICR mice were divided into five groups. Five animals belonged to the no-treatment group, and 15 animals belonged to 
each of the following four groups: O. mykiss-derived PDRN injection (Placentex) group, O. keta-derived PDRN injection (Rejuvenex) group, $O$. keta-derived PDRN cream (Rejuvenex Cream) group, and a $0.9 \%$ normal saline soaked dressing group.

A total of $10 \mathrm{~g}$ of 99\% 2.2.2-tribromoethanol (Purum, SigmaAldrich, St. louis, MO, USA) was first mixed into $10 \mathrm{~mL}$ of tertamyl alcohol (ReagentPlus, Sigma-Aldrich). This 100\% concentration mixture was then mixed with phosphate-buffered saline and diluted to a concentration of 5\%. The diluted mixture was then intraperitoneally injected into the 60 mice in the above four treatment groups, excluding those in the no-treatment group, at a dose of $0.006 \mathrm{~mL} / \mathrm{g}$ for anesthesia. The backs of the mice were subsequently shaved. The back of each mouse was then disinfected with $10 \%$ povidone-iodine and $70 \%$ ethanol. Using a bovie (Megadyne, Draper, UT, USA), a $1.5 \times 1.5 \mathrm{~cm}$-sized full-thickness skin defect was created, which reached the panniculus carnosus and simulated a full-thickness burn. For dressing, Tegaderm (3M, St. Paul, MN, USA) was attached to the defects of all 15 animals in each group, and Medifoam (Mundipharma, Seoul, Korea) was subsequently fixed using Hypafix (BSN Medical, Hamburg, Germany) tape. The experimental materials were administered to the 15 animals in each group every day during wound healing via an intraperitoneal injection or a topical application at a dose of $8 \mathrm{mg} / \mathrm{kg} /$ day $(0.13 \mathrm{~mL}$ for injections and $0.3 \mathrm{~mL}$ for cream and normal saline).

\section{Gross examination and wound size measurement}

A Plastic surgeon (J.H.L.) conducted a gross examination and evaluation of the wounds in terms of the skin necrosis, inflammation, and adverse reactions immediately after the treatment, and again after 4, 7, and 10 days of treatment. A sterilized OHP (overhead projector) film was placed on the wound to trace its boundary; the film was then scanned, and the size of the wound was measured using the Image J Version 1.45 s software (National Institutes of Health, Bethesda, MD, USA). As a control, a 1.5×1.5 $\mathrm{cm}$-sized square was scanned to measure its size. After converting the measured size to $\mathrm{cm}^{2}$, the changes in the size were compared in terms of percentages, where the size measured immediately after the creation of the wound was considered to be $100 \%$. Five ani- mals from each group were sacrificed 4, 7, and 10 days after the creation of wounds. Therefore, on day 4, 15 animals were available for each group; 10 were available in each group on day 7; and five were available on day 10 .

\section{Histological examination}

Tissues were collected from the sacrificed animals, including the entire wound and surrounding wound tissues. The obtained tissues were fixed for more than 6 hours in 10\% formalin. Hematoxylin and eosin staining was subsequently conducted to observe the degree of re-epithelialization, granulation tissue proliferation, necrosis and inflammation, whereas Masson-trichrome staining was conducted to evaluate the formation of collagen. The formation of elastic fibers was used as an index of wound contraction. The skin tissues were embedded in paraffin and sliced at a thickness of 4-6 $\mu \mathrm{m}$. Paraffin was removed from the slices using xylene, and the slices were subsequently dehydrated in alcohol. The Verhoeff reaction was then conducted, and the slices were washed in $2 \%$ ferric chloride aqueous solution for the detection of elastic fibers. Next, the slides were stained with van Gieson solution and examined under an optic microscope.

Using a $\times 40$ magnification optic microscope, 10 fields of view were examined in each tissue slide. The differences among the groups were evaluated by a pathologist. The findings of the wound tissue biopsy, including the extent of re-epithelialization, evidence of granulation tissue proliferation, and inflammation and necrosis of the wound. These values were quantified as described below, and the scores were then summed. The re-epithelialization and granulation tissue proliferation were quantified on a scale of 4 (0-4) as follows: 0 if no re-epithelialization or granulation tissue proliferation was observed under the optic microscope; 1 if it was observed in less than $1 / 4$ of the tissue; 2 if observed in $1 / 4-1 / 2$; 3 if observed in $1 / 2-3 / 4$; and 4 if observed in more than $3 / 4$ of the tissue. Similarly, the inflammation and necrosis of the wound was quantified using scores ranging between -4 and $0 \mathrm{ac}-$ cording to the degree of inflammation and necrosis observed in the wound: a score of 0 was given for the level of inflammation and necrosis observed in normal skin; -1 if colonies of inflammatory cells, including neutrophils, were observed in less than $1 / 4$ of 
the tissue; -2 if observed in $1 / 4-1 / 2 ;-3$ if observed in $1 / 2-3 / 4$; and -4 if observed in more than $3 / 4$ of the tissue (Table 1 ). The scores for re-epithelialization, granulation tissue proliferation, and inflammation and necrosis observed in the stratum corneum and epithelial layer of the wound were summed; and the sums ranged between -4 and 8 , with higher values indicating better wound healing.

\section{Immunohistochemistry}

The tissue samples obtained at 4, 7, and 10 days after wounding were subjected to p63 protein (Santa Cruz Biotechnology, Santa Cruz, CA, USA) staining to measure the formation of epidermis. Further, PECAM/CD31 (Santa Cruz Biotechnology) staining was conducted to assess neovascularization. The tissues were then observed under an optic microscope to comparatively evaluate the proliferation of the epidermis.

The mouse tissues were fixed in formaldehyde, and heat-mediated antigen retrieval was conducted using citrate buffer solution. Subsequently, the samples were blocked for 1 hour in $4 \%$ serum at $22^{\circ} \mathrm{C}$ and were cultured for 15 hours at $4^{\circ} \mathrm{C}$ in a $1 / 40$ dilution containing a primary antibody against the $\mathrm{p} 63$ protein. Biotin-conjugated anti-mouse polyclonal antibodies were used as the secondary antibodies in a 1/250 dilution. Immunostaining was then measured in each sample using an optic microscope and image analyzer; the evaluation of wound healing was conducted by a pathologist.

The tissues embedded in paraffin were sliced at a thickness of 5

Table 1. The quantity analysis of wound healing

$\begin{array}{lccc}\text { Grade } & & & \text { Score } \\ \text { Grade of re- } & \text { Finding of re- } & \text { None } & 0 \\ \text { epithelialization \& } & \text { epithelialization \& } & <1 / 4 & 1 \\ \text { granulation tissue } & \text { granulation tissue } & 1 / 4-1 / 2 & 2 \\ \text { proliferation } & \text { proliferation in wound } & 1 / 2-3 / 4 & 3 \\ & & >3 / 4 & 4 \\ \text { Grade of inflammation \& } & \text { Finding of inflammatory } & \text { None } & 0 \\ \text { necrosis } & \text { cells in wound } & <1 / 4 & -1 \\ & & 1 / 4-1 / 2 & -2 \\ & & 1 / 2-3 / 4 & -3 \\ & & >3 / 4 & -4\end{array}$

Using a $\times 40$ magnification optic microscope, 10 fields of view were examined in each tissue slide. The scores for re-epithelialization, granulation tissue proliferation, and inflammation and necrosis observed in the stratum corneum and epithelial layer of the wound were summed; and the sums ranged between -4 and 8 , with higher values indicating better wound healing. $\mathrm{mm}$, and antigen retrieval was conducted using $0.05 \mathrm{M}$ sodium citrate buffer. The tissues were treated with a primary antibody against platelet endothelial cell adhesion molecule-1 (PECAM-1)/ CD31 (Santa Cruz Biotechnology). The secondary antibody was provided by Innovex (Richmond, CA, USA), and the reaction was visualized using 3,30-diaminobenzidine tetrahydrochloride (Sigma-Aldrich). The slides were counter-stained with hematoxylin and cover slipped.

\section{Enzyme-linked immunosorbent assays}

Tissues were obtained from the sacrificed animals including from the entire wound and surrounding wound tissues, and enzymelinked immunosorbent assays (ELISA) were conducted to measure the vascular endothelial growth factor (VEGF) and transforming growth factor- $\beta$ (TGF- $\beta$ ) levels. A total of $0.1 \mathrm{~g}$ of the skin tissue was homogenized in $150 \mu \mathrm{L}$ of radioimmunoprecipitation assay lysis buffer containing protease inhibitors (50 mM Tris, $\mathrm{pH}$ 8.0; $150 \mathrm{mM} \mathrm{NaCl} ; 1 \%$ Triton X-100; 0.5\% sodium deoxycholate; $0.1 \%$ sodium dodecyl sulfate; $100 \mathrm{mM}$ phenylmethylsulfonyl fluoride; and $1.0 \mathrm{mg}$ aprotinin $/ \mathrm{mL}$ ) and incubated for 30 minutes at $4^{\circ} \mathrm{C}$. The sample was then centrifuged at $10,000 \times g$ at $4{ }^{\circ} \mathrm{C}$ for 15 minutes and the supernatant was stored at $-70^{\circ} \mathrm{C}$ until use.

In order to measure angiogenesis, which exerts a significant influence on wound healing, VEGF mouse ELISA kits (Abcam Inc., Cambridge, MA, USA) were used to compare the various groups. In order to measure the TGF- $\beta$ and fibronectin levels, which are considered indicators of inflammation in white mice, TGF- $\beta 1$ mouse ELISA kits (Abcam Inc.) were used to compare the expression among the experimental groups.

\section{Statistical analysis}

Statistical analyses were conducted using the IBM SPSS ver. 20.0 (IBM Corp., Armonk, NY, USA). Tendencies among the data were identified and analyzed using a table where measurements were sorted according to the time, stage, and figure for each group, and are shown as the mean \pm standard error of the mean. To compare the changes in wound size observed each day in each treatment group, two-way analysis of variance (ANOVA) was conducted using SPSS, and Bonferroni tests were carried out as 
post-hoc tests. Regarding the findings obtained from the wound tissue biopsy in each treatment group, Kruskal-Wallis tests, which are non-parametric, were performed due to the small sample sizes. The $p$-values were obtained from Mann-Whitney U-tests conducted as post-hoc tests. Differences between the groups were deemed significant if $p$-value was less than 0.05 ; such differences were indicated as $p<0.05$.

\section{RESULTS}

\section{Gross examination}

In all experimental groups, the wound healed with time. The wounds healed more rapidly in the PDRN injection and cream groups. When the PDRN-based treatment groups were compared with the control group before the treatment and after 4, 7, and 10 days of treatment, no delayed responses in wound healing, including skin necrosis, inflammation, or other adverse reactions, were seen in any of the groups. In the O. keta-derived PDRN cream group, the topically-applied cream remained on the wound, and the wound thus appeared as if it had impurities and was less clean when compared with that reported for other groups. However, when the cream was removed, wound healing could be seen from inside of the wound (Fig. 1).

\section{Wound size measurement}

In all PDRN-based treatment groups, the wound size decreased with time. In comparison with that for the control group (normal saline soaked dressing group), the wound size decreased more rapidly in the PDRN injection and cream groups. The differences between each PDRN-based treatment group and the control group on days 4, 7, and 10 were also significant (two-way ANOVA, $p<0.05)$. Post-hoc analyses of the differences between the PDRN treatment groups on different days further indicated that the $O$. keta-derived PDRN injection had significantly better wound healing effects than those of normal saline soaked dressing, as evidenced by the changes in wound size observed at all time points (two-way ANOVA, $p<0.015$ ). A similar observation was made for

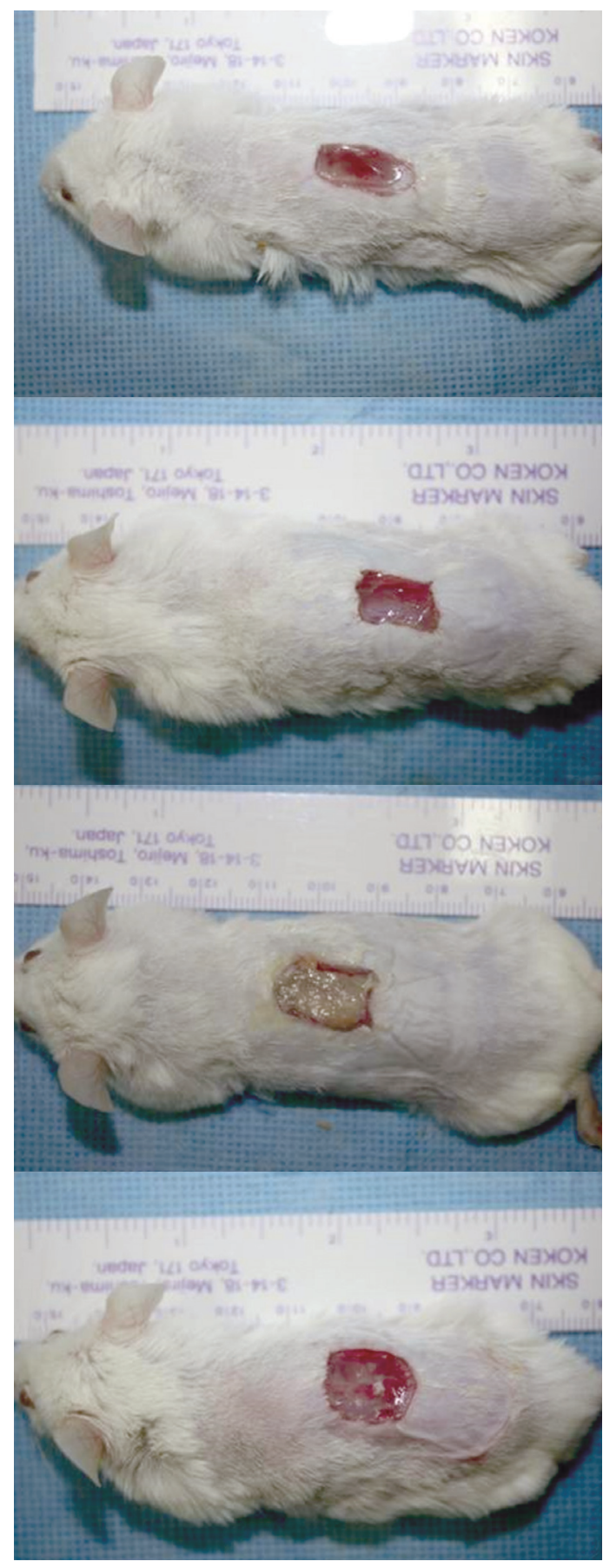

Fig. 1. Gross findings of the full-thickness wound on the dorsum of the mouse on days 10: the Oncorhynchus mykiss-derived polydeoxyribonucleotide (PDRN) injection group, the Oncorhynchus ketaderived PDRN injection group, the $O$. keta-derived PDRN cream group, the normal saline soaked dressing group, respectively. There was no delayed wound healing response in all the groups across all time periods. In the $O$. keta-derived PDRN cream group, the wound appeared as if it had impurities and was less clean when compared with the other groups. However, when the cream was removed, wound healing could be seen from inside of the wound. 
the O. mykiss-derived PDRN injection group. For the O. keta-derived cream, the wound size decreased more rapidly at all time points compared to that observed for the normal saline soaked dressing group, suggesting better wound healing. However, no statistically significant increase was observed. The order of efficacy of the PDRN-based treatment and control groups in terms of the wound healing measured on days 4, 7, and 10 was as follows: O. mykiss-derived PDRN injection, $O$. keta-derived PDRN injection, $O$. keta-derived PDRN cream, and normal saline soaked dressing. However, no statistically significant difference was found among the PDRN-based treatment groups (Fig. 2).

\section{Histological examination}

\section{Hematoxylin and eosin staining}

The degree of wound healing was compared using scores that quantified the amount of re-epithelialization, granulation tissue proliferation, and inflammation and necrosis of the wound. All PDRN-based treatment groups were characterized by clear re-epithelialization and granulation tissue proliferation in comparison with that observed for the normal saline soaked group at all time points (days 4, 7, and 10); moreover, the infiltration of inflammatory cells decreased in the PDRN-based treatment groups (Fig. 3). Over time, the scores for the degree of wound healing increased in all experimental groups. When the scores were compared, significant differences among the experimental groups were observed on days 4 and 7 (Kruskal-Wallis test, $p<0.005$ ), although no significant difference was seen on day 10 (Kruskal-Wallis test, $p=0.09$ ). When post-hoc analyses were conducted to compare the experimental groups, the PDRN-based treatment groups exhibited significantly better wound healing effects in comparison with that reported for the control group on days 4 and 7 (Mann-Whitney test, $p<0.05)$. On day 4 , no difference was observed between the O. mykiss-derived PDRN injection and O. keta-derived

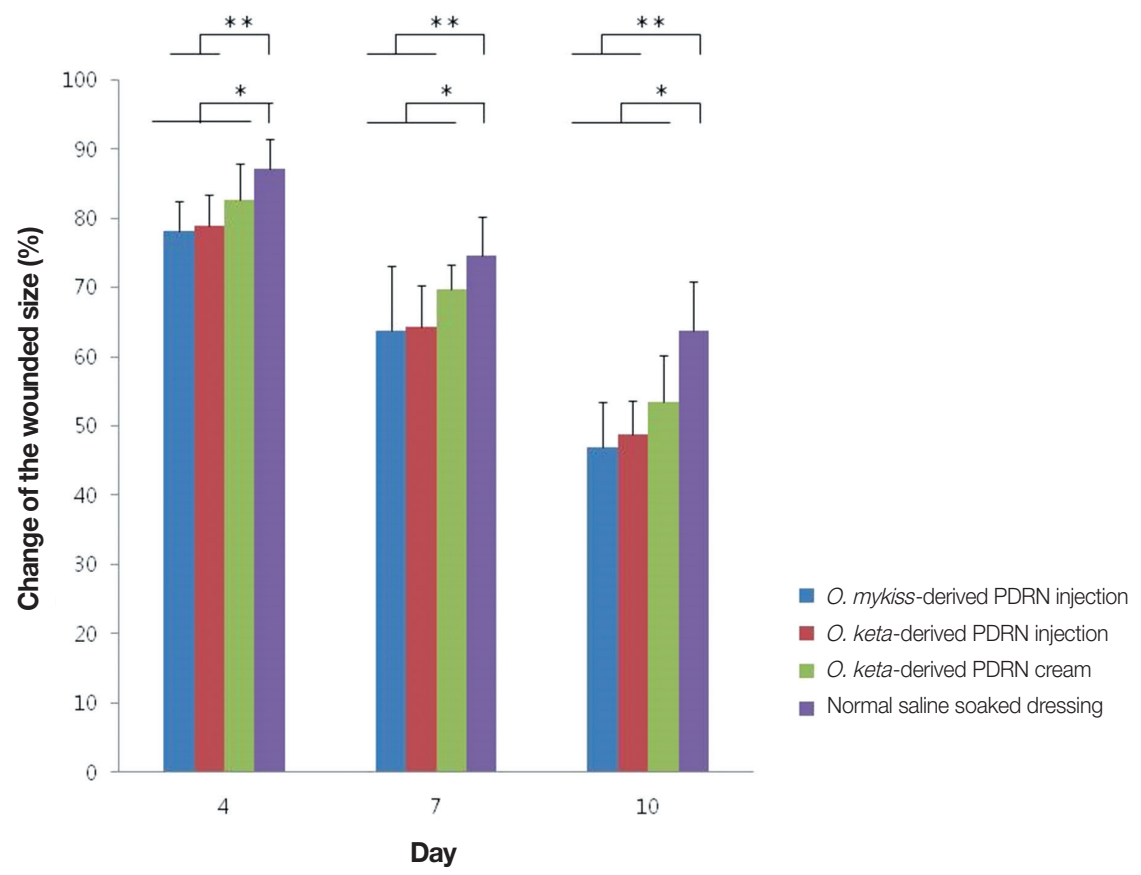

Fig. 2. The changes of the wound size (\%). In all polydeoxyribonucleotide (PDRN)-based treatment groups, the wound size decreased with time. In comparison with that for the normal saline soaked dressing group, the wound size decreased more rapidly in the PDRN-based treatment groups. The order of efficacy of the PDRN-based treatment and control groups in terms of the wound healing measured on days 4, 7, and 10 was as follows: $O$. mykiss-derived PDRN injection, $O$. keta-derived PDRN injection, $O$. keta-derived PDRN cream, and normal saline soaked dressing. All values are expressed as mean \pm standard deviation from four different independent experimental sets. Significant differences are indicated by $^{*} p<0.05$ and ${ }^{* *} p<0.015$. O, Oncorhynchus. 

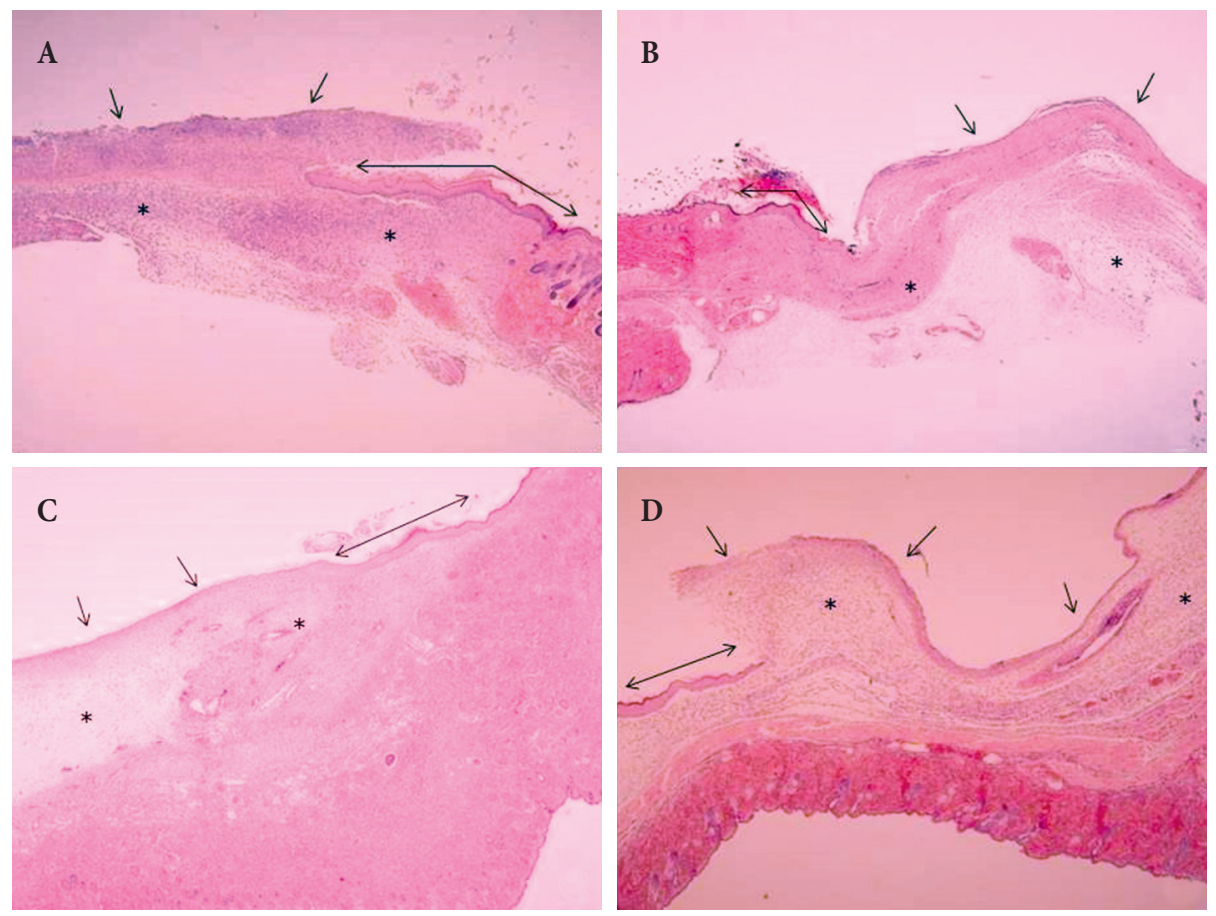

Fig. 3. Histological findings of the wound $(\mathrm{H} \& \mathrm{E}, \times 40)$. On day 10 , (A) the Oncorhynchus mykiss-derived polydeoxyribonucleotide (PDRN) injection group, (B) the Oncorhynchus keta-derived PDRN injection group, (C) the O. keta-derived PDRN cream group, (D) normal saline soaked dressing group. Areas of granulation tissue proliferation are marked with asterisks $(*)$, areas of re-epithelialization $(\leftrightarrow)$, and sites coated with inflammatory cells $(\uparrow)$. All PDRN-based treatment groups were characterized by clear re-epithelialization and granulation tissue proliferation in comparison with that observed for the normal saline soaked dressing group at all time points (days 4, 7, and 10); moreover, the infiltration of inflammatory cells decreased in the PDRN-based treatment groups.

PDRN cream groups; however, a significant difference was found between the $O$. keta-derived PDRN injection and cream groups (Mann-Whitney test, $p<0.05$ ). The wound healing effects were the greatest in the $O$. keta-derived PDRN injection and $O$. mykiss-derived PDRN injection groups, which showed similar scores, followed by the $O$. keta-derived cream and normal saline soaked dressing groups (Fig. 4).

\section{Masson's trichrome staining}

No collagen formation was initially observed in any of the groups. At all time points, pronounced collagen formation was observed in the PDRN-based treatment groups in comparison with that in the normal saline soaked dressing group (Fig. 5). When the scores for collagen formation were compared, no significant difference was found among the groups on days 4 and 7 . On day 4 , the scores for collagen formation in all experimental groups were 0 . On day 10 , collagen formation was observed in all experimental groups, but there were significant differences among them (Kruskal-Wallis test, $p<0.05)$. Post-hoc analyses showed that the collagen formation had increased more significantly in the $O$. keta-derived PDRN injection group than in the normal saline soaked dressing group (Mann-Whitney test, $p<0.05$ ). The effects of the $O$. keta-derived PDRN cream, which contains less PDRN, were smaller than those of the PDRN injections (Fig. 6).

\section{Elastin staining}

The formation of elastic fibers was observed in order to measure wound contraction in the collected tissue samples. Although multiple tests were repeated, all experimental groups yielded similar results; at all time points, elastic fiber formation was similarly weak in all experimental groups (Figs. 7, 8). 


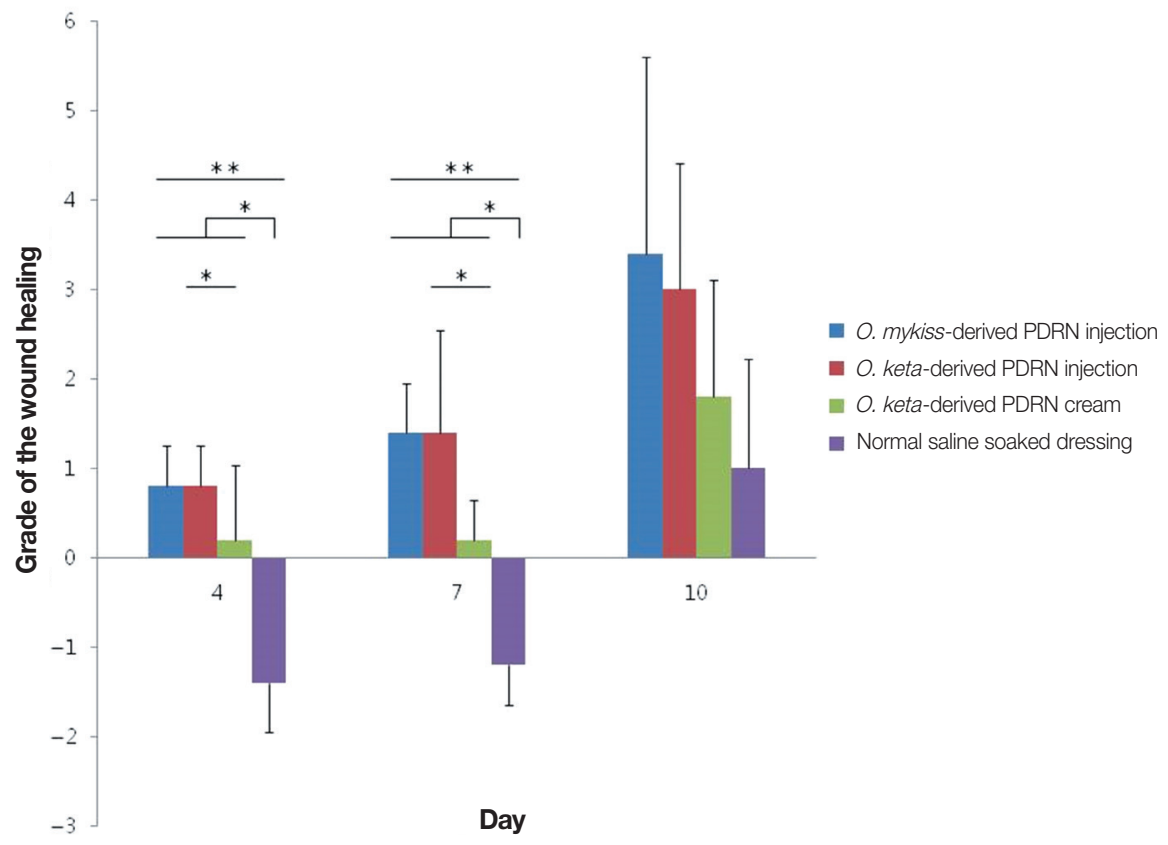

Fig. 4. The grades of the wound healing. Over time, the scores for the degree of wound healing increased in all experimental groups. The wound healing effects were the greatest in the O. keta-derived polydeoxyribonucleotide (PDRN) injection and O. mykiss-derived PDRN injection groups, which showed similar scores, followed by the $O$. keta-derived cream and normal saline soaked dressing groups. All values are expressed as mean \pm standard deviation from four different independent experimental sets. Significant differences are indicated by ${ }^{\star} p<0.05$ and ${ }^{* *} p<0.005$. O, Oncorhynchus .
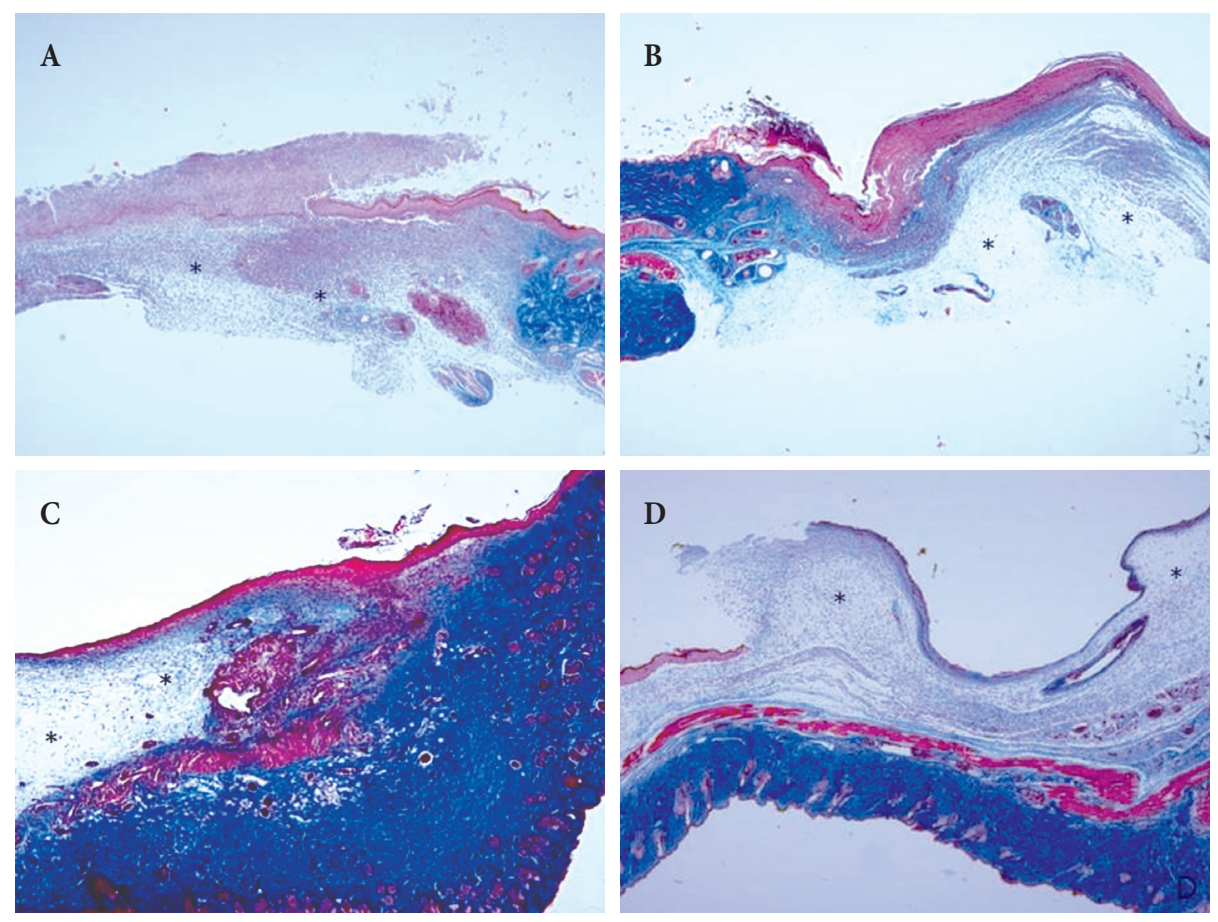

Fig. 5. Histological findings of the wound (Masson's trichrome stain, $\times 40$ ). On day 10 , (A) the Oncorhynchus mykiss-derived polydeoxyribonucleotide (PDRN) injection group, (B) the Oncorhynchus keta-derived PDRN injection group, (C) the O. keta-derived PDRN cream group, (D) the normal saline soaked dressing group. Asterisks $\left(^{*}\right)$ indicate areas of granulation tissue proliferation. Collagen is the blue-colored cells in the area where the granulation tissue is proliferated. At all time points (days 4, 7, and 10), pronounced collagen formation was observed in the PDRNbased treatment groups in comparison with that in the normal saline soaked group. 


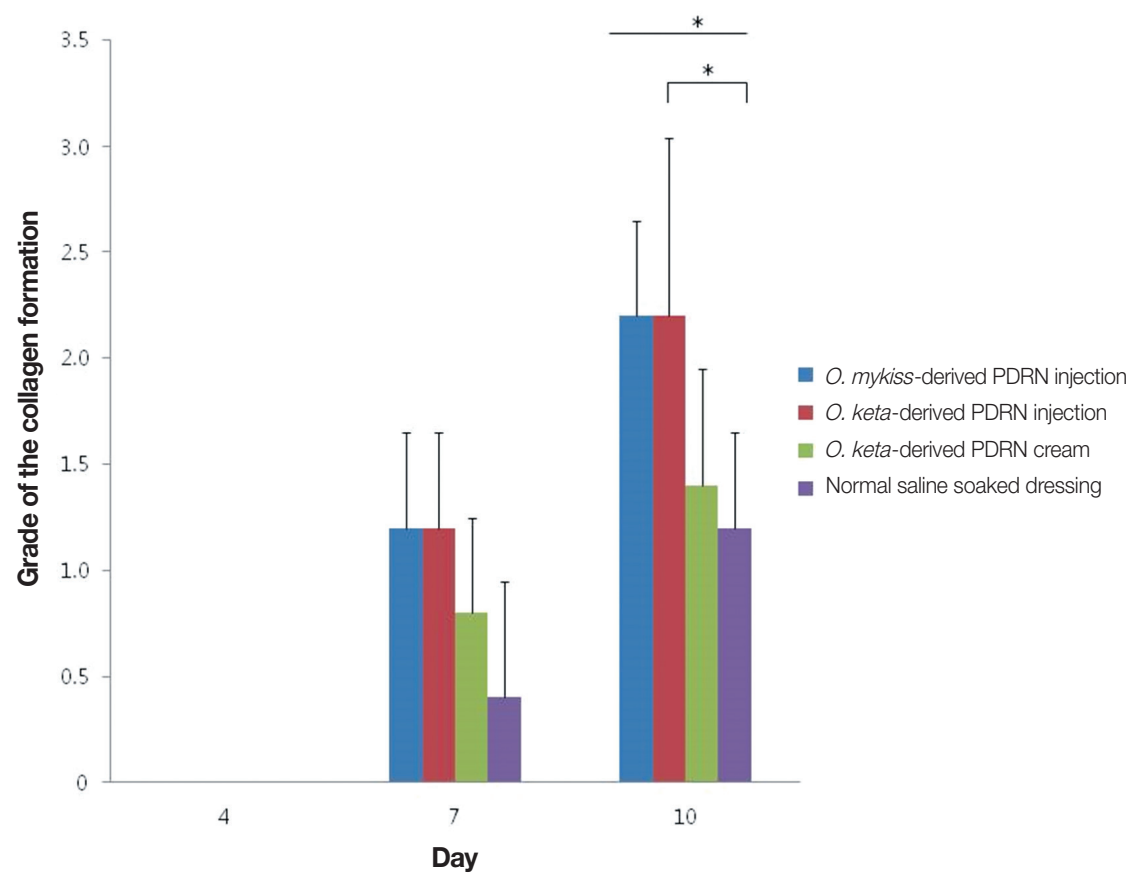

Fig. 6. The grades of the collagen formation. When the scores for collagen formation were compared, no significant difference was found among the groups on days 4 and 7. On day 4 , the scores for collagen formation in all experimental groups were 0 . On day 10 , collagen formation was observed in all experimental groups, but there were significant differences among them. The collagen formation had increased more significantly in the $O$. keta-derived polydeoxyribonucleotide (PDRN) injection group than in the normal saline soaked dressing group. All values are expressed as mean \pm standard deviation from four different independent experimental sets. Significant differences are indicated by ${ }^{\star} p<0.05$. O, Oncorhynchus .

\section{Immunohistochemistry}

p63

When the expression of the p63 protein, which is involved in epidermal development, was observed in the collected tissue samples, no significant differences were noted on days 4 and 7. However, significant differences between the PDRN-based treatment and normal saline soaked dressing groups and between the PDRN injection and PDRN cream groups were seen on day 10 (Kruskal-Wallis test, $p<0.02$ ). Moreover, post-hoc analyses of the expression of p63 in each group indicated that the expression was higher in the PDRN injection groups than in the PDRN cream and normal saline soaked dressing groups at all times. On day 10, the expression was significantly higher in the PDRN injection groups than in the normal saline soaked dressing group (Mann-Whitney test, $p<0.01$ ). The expression was the highest in the $O$. keta-derived PDRN injection and $O$. mykiss-derived PDRN injection groups, which showed similar levels, followed by the $O$. keta-derived PDRN cream group and the normal saline soaked dressing group (Fig. 9).

\section{CD31}

When PECAM/CD31 staining was conducted to evaluate the neovascularization in the collected tissues, no significant differences were observed among the experimental groups at any of the time points. Post-hoc analyses showed that the amount of neovascularization was higher in the PDRN-base treatment groups in comparison with that in the control group on days 7 and 10; however, no statistical significance was noted (Fig. 10).

\section{Enzyme-linked immunosorbent assays}

\section{Vascular endothelial growth factor}

When measurements of angiogenesis, which is crucial for wound healing, were made and compared using VEGF mouse ELISA kits (Abcam Inc.), significant differences were observed among the experimental groups on days 4 and 7 (Kruskal-Wallis test, $p<0.05)$. Post-hoc analyses of the VEGF expression in each group showed that the expression was higher in the PDRN injection groups than in the PDRN cream and normal saline soaked dress- 

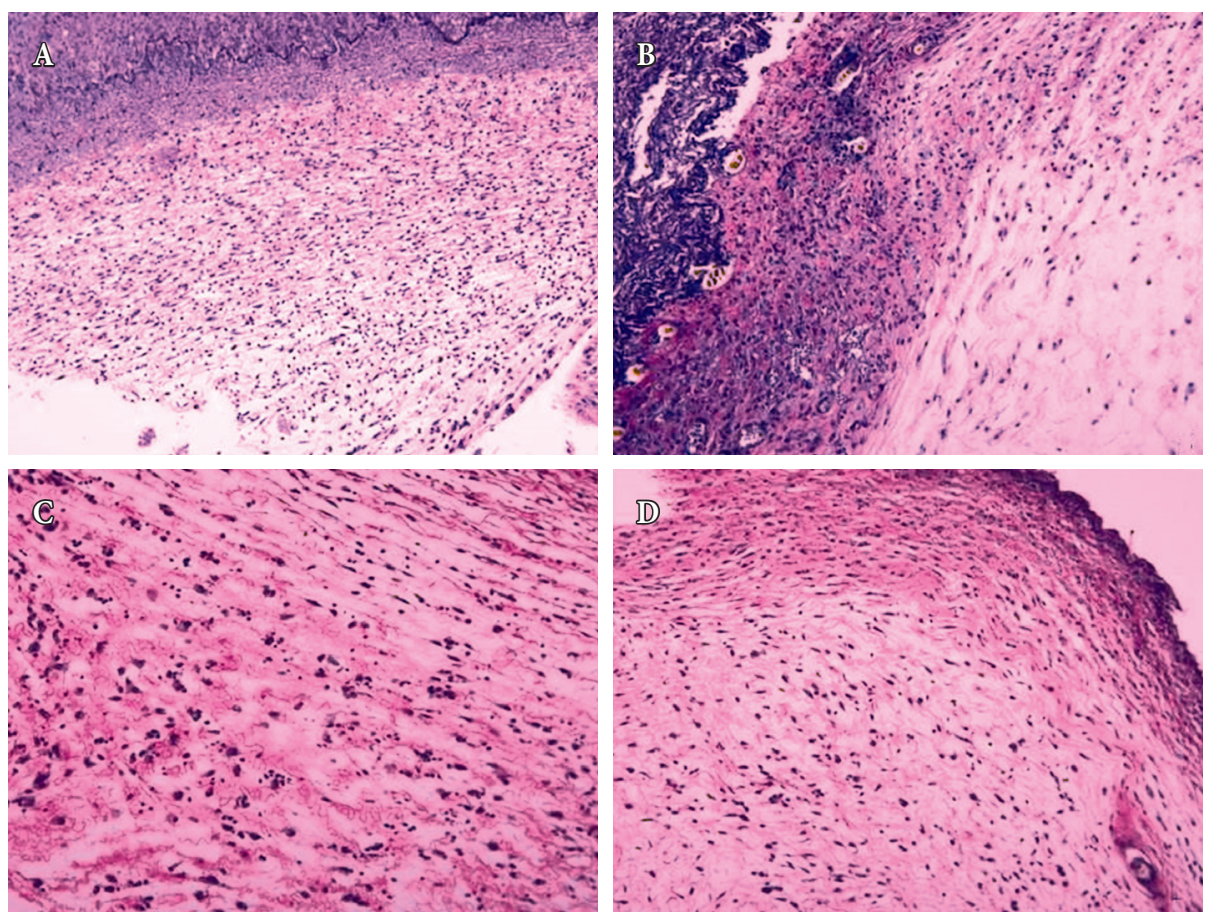

Fig. 7. Histological findings of the wound (Elastin stain, $\times 200$ ). On day 10, (A) the Oncorhynchus mykiss-derived polydeoxyribonucleotide (PDRN) injection group, (B) the Oncorhynchus keta-derived PDRN injection group, (C) the O. keta-derived PDRN cream group, (D) the normal saline soaked dressing group. Elastic fiber is the black-colored cells in the area where the granulation tissue is proliferated. At all-time points (days 4,7 , and 10), elastic fiber formation was similarly weak in all experimental groups.

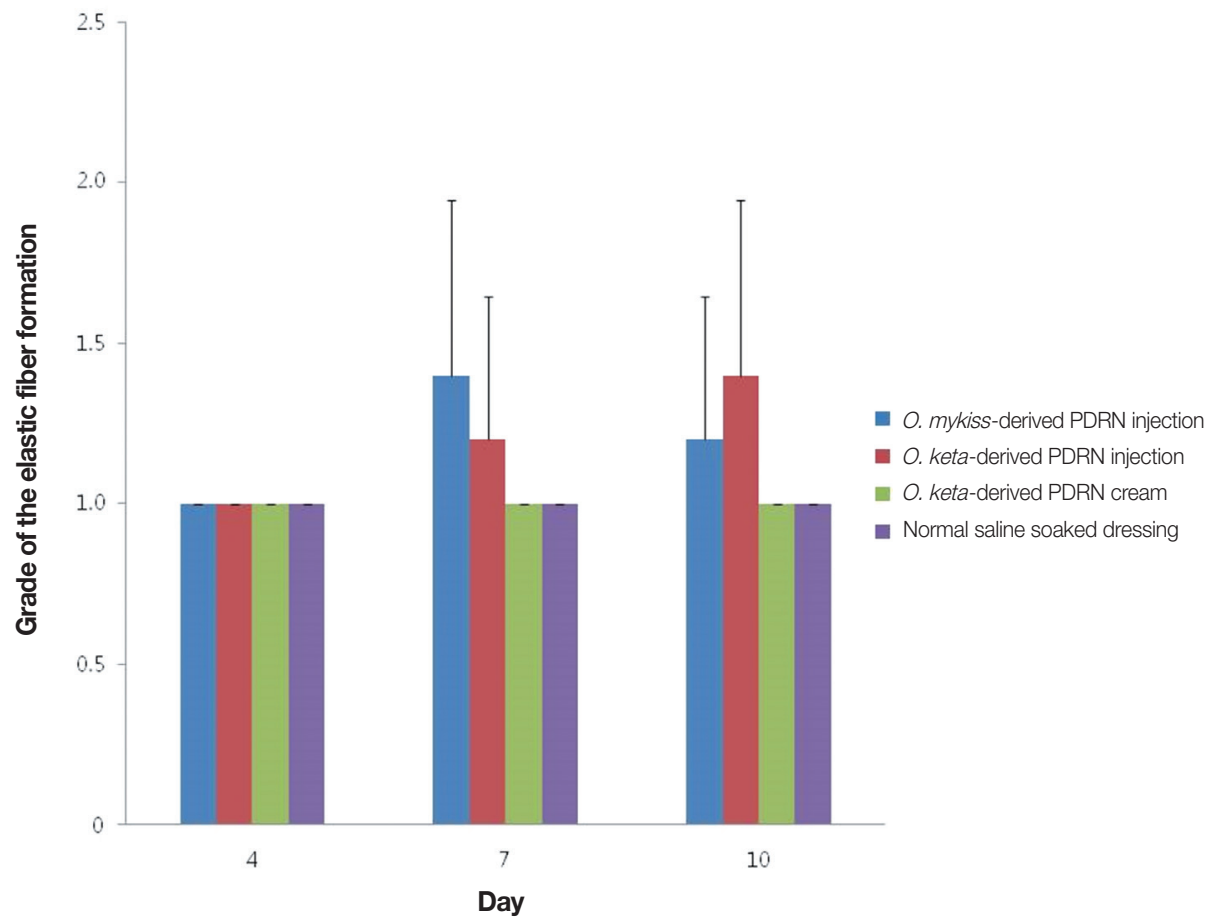

Fig. 8. The grades of the elastic fiber formation. All experimental groups yielded similar results. All values are presented as mean \pm standard deviation from four different independent experimental sets. O, Oncorhynchus; PDRN, polydeoxyribonucleotide. 


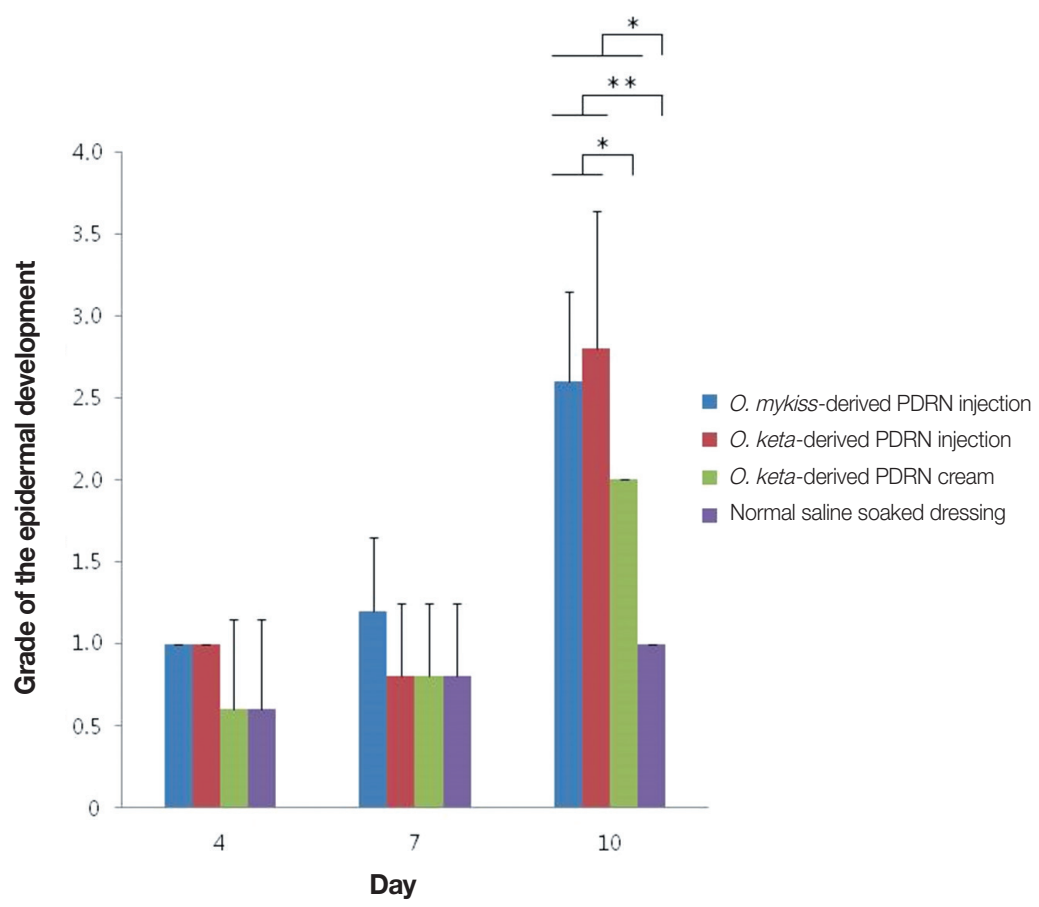

Fig. 9. The grades of the epidermal development by expression of p63. There were no significant differences on days 4 and 7. However, significant differences between the polydeoxyribonucleotide (PDRN)-based treatment and normal saline soaked dressing groups and between the PDRN injection and PDRN cream groups were seen on day 10. On day 10, the expression was significantly higher in the PDRN injection groups than in the normal saline soaked dressing group. All values are presented as mean \pm standard deviation from four different independent experimental sets. Significant differences are indicated by ${ }^{\star} p<0.02$ and ${ }^{* *} p<0.01$. O, Oncorhynchus.

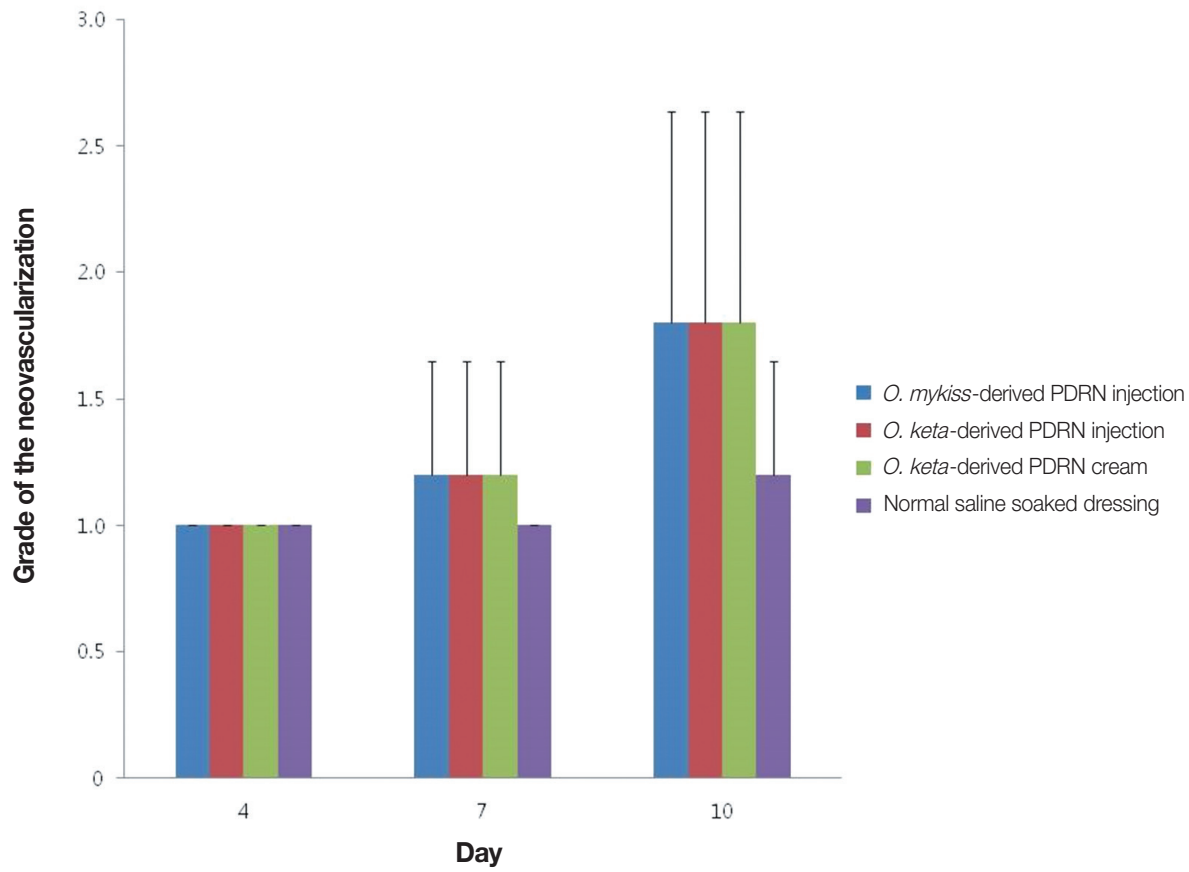

Fig. 10. The grades of the neovascularization by the expression of CD31. There were no significant differences among the experimental groups at any of the time points. The amount of neovascularization was higher in the polydeoxyribonucleotide (PDRN)-base treatment groups in comparison with that in the control group on days 7 and 10 ; however, no statistical significance was noted. All values are presented as mean \pm standard deviation from four different independent experimental sets. O, Oncorhynchus. 
ing groups at all time points. Nevertheless, no statistically significant difference was observed. VEGF expression in the PDRN injection groups was the highest on day 7 and decreased on day 10. The expression was the highest in the O. mykiss-derived PDRN injection group, followed by that in the $O$. keta-derived PDRN injection group, $O$. keta-derived PDRN cream group, then the normal saline soaked dressing group. Although the increase in VEGF was slower in the $O$. keta-derived PDRN cream group than in the PDRN injection group, it showed a tendency to increase with time. This is illustrated by the fact that the VEGF expression in this group was higher than that of the normal saline soaked dressing group on day 10 (Fig. 11).

\section{Transforming growth factor- $\beta$}

When measurements of TGF- $\beta$ and fibronectin, which are indicators of inflammation in white mice, were made using TGF- $\beta 1$ mouse ELISA kits (Abcam Inc.), significant differences were observed among the groups at all time points (Kruskal-Wallis test, $p<0.02)$. Post-hoc analyses of the TGF- $\beta$ levels in each experimen- tal group showed that the levels were higher in the PDRN-based treatment groups than in the normal saline soaked dressing group at all time points (Mann-Whitney test, $p<0.02$ ). In the $O$. keta-derived PDRN injection group, the TGF- $\beta$ level was the highest on day 7 and decreased on day 10. The level observed in the $O$. keta-derived PDRN injection group on day 7 was similar to that found in the O. mykiss-derived PDRN injection group. Although the effects of the $O$. keta-derived PDRN cream were somewhat weaker compared to those of the PDRN injection group, the expression of TGF- $\beta$ still increased in comparison to that observed in the normal saline soaked dressing group. The expression of TGF- $\beta$ was highest in the $O$. mykiss-derived PDRN injection group, followed by the $O$. keta-derived PDRN injection group, the $O$. keta-derived PDRN cream group, then the normal saline soaked dressing group (Fig. 12).

\section{DISCUSSION}

Various dressings are being used to treat excisional wounds of the

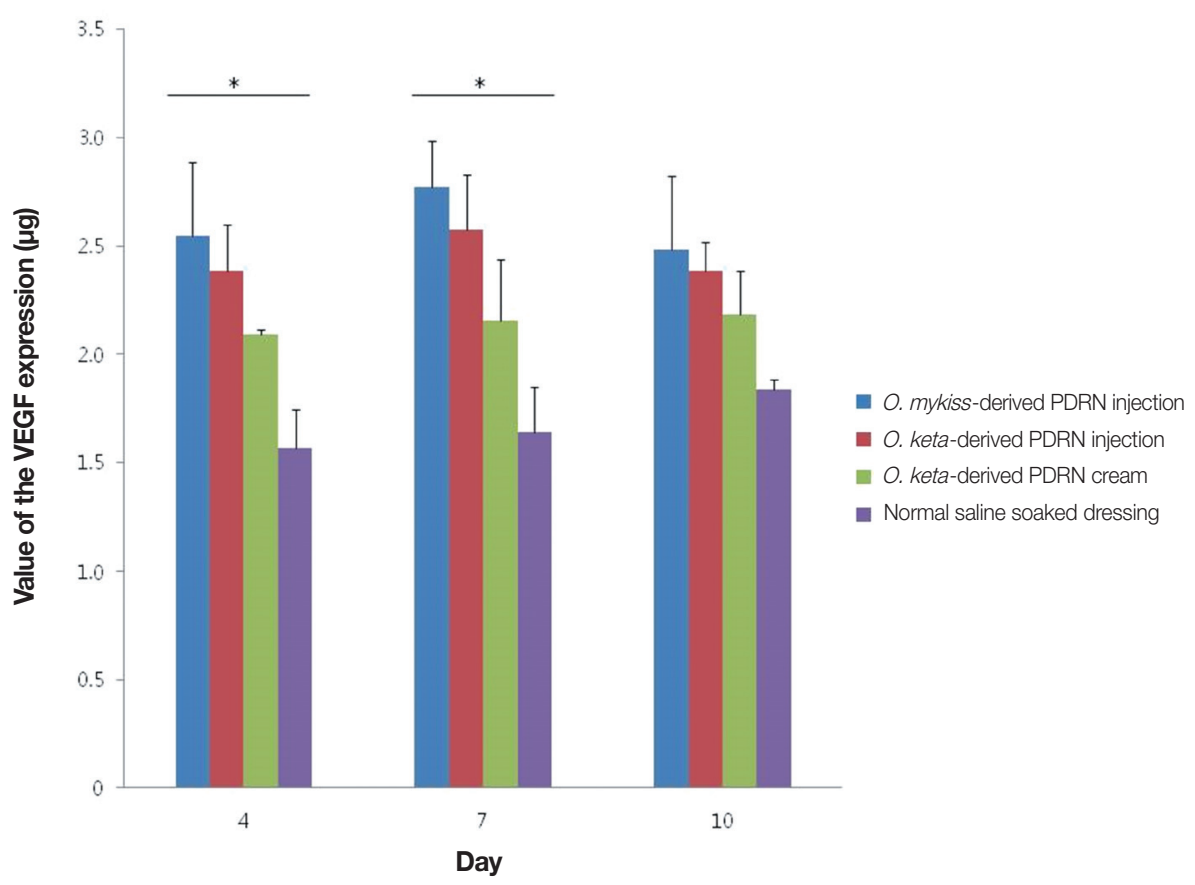

Fig. 11. The grades of angiogenesis by the values of the vascular endothelial growth factor (VEGF) expression. The expression was the highest in the O. mykiss-derived polydeoxyribonucleotide (PDRN) injection group, followed by that in the O. keta-derived PDRN injection group, O. ketaderived PDRN cream group, then the normal saline soaked dressing group. There were significant differences among the experimental groups on days 4 and 7. All values are presented as mean \pm standard deviation from four different independent experimental sets. Significant differences are indicated by ${ }^{*} p<0.05$. O, Oncorhynchus. 
skin caused by trauma or burns. Recently, dressings that utilize growth factors, including PDGF (becaplermin), epidermal growth factor, and basic fibroblast growth factor, have been developed and used. Multiple studies have reported therapeutic roles for adenosine and $\mathrm{A} 2 \mathrm{~A}$ receptor agonists in promoting wound healing [14-16]. Adenosine A2A receptors, belonging to the P1 subtype of purinergic receptors, are expressed in most cell types involved in wound healing, including macrophages, fibroblasts, and endothelial cells. In these cells, activation of A2A reduces inflammation, promotes the proliferation of endothelial cells and fibroblasts, increases collagen synthesis and the proportion of granulation tissue, and stimulates the secretion of VEGF, ultimately promoting wound healing $[10,12,17,18]$.

PDRN, an $\mathrm{A} 2 \mathrm{~A}$ receptor agonist, is a polymer in which purine and pyrimidine nucleotides have bonded together through phosphodiester bonds, and is reported to mostly influence nucleotides. Such nucleotides and nucleosides promote the growth of various cell types while also stimulating the synthesis of nucleic acids and wound healing, by stimulating salvage pathways, which produce nucleic acids at a low energy expenditure, and by activating A2A purinergic receptors $[18,19]$.

Studies investigating the safety of PDRN, particularly its acute and chronic toxicity, have confirmed through gross and histological examinations that PDRN does not result in any mortality or have any toxic effect on the liver, lungs, brain, skeletal muscles, or heart $[11,20]$. The $O$. mykiss-derived PDRN injection used in the present study has been clinically approved and is used in Italy as a local and non-oral drug [6]. Several studies have reported that the injection of PDRN promotes the proliferation of human fibroblasts and osteoblasts in in vitro models $[8,9,12]$. In animal experiments, intraperitoneal injections of PDRN were observed to have beneficial effects in a rat model of ischemic skin flaps [21]. Moreover, intraperitoneal injections of PDRN were also used in diabetic mice to stimulate angiogenesis and promote wound healing [10]. In addition, in mice with deep dermal second-degree burns, intraperitoneal PDRN injections were reported to improve wound

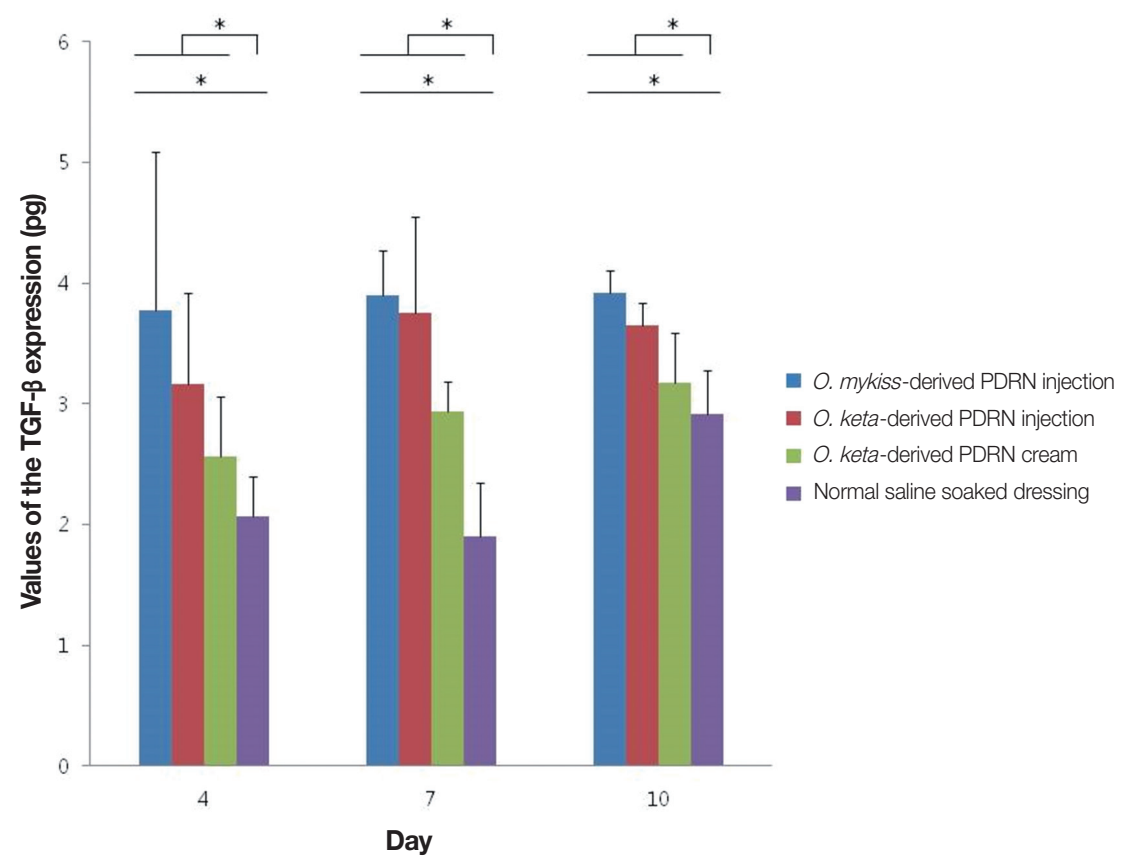

Fig. 12. The levels of the transforming growth factor- $\beta$ (TGF- $\beta$ ) expression. There were significant differences among the experimental groups at all time points. The expression of TGF- $\beta$ was highest in the $O$. mykiss-derived PDRN injection group, followed by the $O$. keta-derived polydeoxyribonucleotide (PDRN) injection group, the $O$. keta-derived PDRN cream group, then the normal saline soaked dressing group. The TGF- $\beta$ levels were higher in the PDRN-based treatment groups than in the normal saline soaked dressing group at all time points. All values are presented as mean \pm standard deviation from four different independent experimental sets. Significant differences are indicated by ${ }^{*} p<0.02 . O, O n c o r h y n c h u s$. 
healing [11]. Clinically, wound healing was promoted when PDRN was injected into the muscle and tissues surrounding wounds at skin transplantation donor sites [22]. In patients with pressure sores, injecting PDRN into the muscle and tissues around the sore was reported to promote healing and to decrease the need for additional treatment as well as the length of the hospital stay [20].

The present study compared the treatment effects of $O$. mykissderived PDRN injection, $O$. keta-derived PDRN injection, $O$. keta-derived PDRN cream, and 0.9\% normal saline soaked dressing on a full-thickness skin defect wound on the backs of ICR mice. The changes in the wounds were observed through gross examination and biopsy after 4, 7, and 10 days. In particular, tissue staining (hematoxylin and eosin staining, Masson trichrome staining, and elastin staining), immunostaining (p63 and CD31 stain), and ELISA (VEGF and TGF- $\beta$ tests) were conducted on the biopsy samples, and the results were analyzed.

According to the gross examinations and an evaluation of the changes in wound sizes, there was no delayed response in wound healing, including skin necrosis, inflammation, or other adverse reactions, observed in any of the groups. However, considering that Tegaderm was fixed on the wound immediately after it creation in order to reduce variations in wound contraction resulting from the dressing, it was interpreted that the formation of the epidermis would tend to be delayed compared to wounds without the Tegaderm $[23,24]$.

Nonetheless, in terms of the changes in wound sizes, marked decreases in size were observed in the $O$. keta-derived PDRN injection and $O$. mykiss-derived PDRN injection groups when compared to the normal saline soaked dressing group. The wound healing effects of the $O$. keta-derived PDRN injection did not differ significantly from those of the O. mykiss-derived PDRN injection. The $O$. keta-derived PDRN cream group also showed more pronounced would healing effects in comparison to the normal saline soaked dressing group, even though the effects were not as great as those of the PDRN injections. If the content of PDRN could be increased in the PDRN cream, it is likely that the effects would be similar to those observed following PDRN injection.

When the biopsy findings were evaluated, the $O$. keta-derived PDRN injection and O. mykiss-derived PDRN injection groups were found to have more active wound healing and collagen formation than the normal saline soaked dressing group at all time points, as evidenced by re-epithelialization, granulation tissue proliferation, inflammatory responses, and neovascularization; in other words, the $O$. keta-derived PDRN injection was effective in promoting wound contraction and healing.

The elastic fiber formation observed by elastin staining was similar in all groups. Based on the above findings, it is speculated that the $O$. keta-derived PDRN injection maintain the normal wound healing process while also promoting wound healing.

ELISA was conducted to compare the TGF and VEGF levels, markers of inflammation and neovascularization, respectively. The levels of both markers in the $O$. keta-derived PDRN injection and O. mykiss-derived PDRN injection groups increased at all time points compared to that at baseline (days 4, 7, and 10); this finding implies that the $O$. keta-derived PDRN injection has similar wound healing-promoting effects as the O. mykiss-derived PDRN injection. Moreover, although the effects of the PDRN cream were not as great as those of injections, the cream was still more effective than normal saline soaked dressing.

When the wound healing effects were analyzed 4, 7, and 10 days after the creation of full-thickness skin defect wounds on the backs of ICR mice, the $O$. keta-derived PDRN injection and PDRN cream were both found to be more effective than normal saline soaked dressing. Moreover, since the wound healing effects of the $O$. keta-derived PDRN injection did not reveal any significant difference compared to those of the $O$. mykiss-derived injection, which is currently approved for clinical use, the $O$. keta-derived PDRN injection is thought to be effective in promoting wound healing for full-thickness skin defects.

However, a limitation of the present study is that it was conducted in animals; in order to apply the findings in the clinical setting, multiple variables regarding wounds, including the size and local condition around the wounds and the systemic condition of the patients, should be considered. Thus, future studies should investigate the mechanisms of action of $O$. keta-derived PDRN and its safety and efficacy should be evaluated in large randomized clinical trials. 


\section{CONFLICT OF INTEREST}

No potential conflict of interest relevant to this article was reported.

\section{REFERENCES}

1. Hunt TK, Hopf H, Hussain Z. Physiology of wound healing. Adv Skin Wound Care 2000;13(2 Suppl):6-11.

2. Cha J, Falanga V. Stem cells in cutaneous wound healing. Clin Dermatol 2007;25:73-8.

3. Valls MD, Cronstein BN, Montesinos MC. Adenosine receptor agonists for promotion of dermal wound healing. Biochem Pharmacol 2009;77:1117-24.

4. Montesinos MC, Gadangi P, Longaker M, Sung J, Levine J, Nilsen D, et al. Wound healing is accelerated by agonists of adenosine A2 ( $\mathrm{G}$ alpha s-linked) receptors. J Exp Med 1997;186:1615-20.

5. Tonello G, Daglio M, Zaccarelli N, Sottofattori E, Mazzei M, Balbi A. Characterization and quantitation of the active polynucleotide fraction (PDRN) from human placenta, a tissue repair stimulating agent. J Pharm Biomed Anal 1996;14:1555-60.

6. Bitto A, Polito F, Altavilla D, Minutoli L, Migliorato A, Squadrito F. Polydeoxyribonucleotide (PDRN) restores blood flow in an experimental model of peripheral artery occlusive disease. J Vasc Surg 2008;48:1292-300.

7. Bianchini P, Tellini N, Morani AM, Folloni MG. Pharmacological data on polydeoxyribonucleotide of human placenta. Int J Tissue React 1981;3:151-4.

8. Guizzardi S, Galli C, Govoni P, Boratto R, Cattarini G, Martini D, et al. Polydeoxyribonucleotide (PDRN) promotes human osteoblast proliferation: a new proposal for bone tissue repair. Life Sci 2003;73:1973-83.

9. Sini P, Denti A, Cattarini G, Daglio M, Tira ME, Balduini C. Effect of polydeoxyribonucleotides on human fibroblasts in primary culture. Cell Biochem Funct 1999;17:107-14.

10. Galeano M, Bitto A, Altavilla D, Minutoli L, Polito F, Calo M, et al. Polydeoxyribonucleotide stimulates angiogenesis and wound healing in the genetically diabetic mouse. Wound Repair Regen 2008;16:20817.

11. Bitto A, Galeano M, Squadrito F, Minutoli L, Polito F, Dye JF, et al. Polydeoxyribonucleotide improves angiogenesis and wound healing in experimental thermal injury. Crit Care Med 2008;36:1594-602.

12. Thellung S, Florio T, Maragliano A, Cattarini G, Schettini G. Polydeoxyribonucleotides enhance the proliferation of human skin fibroblasts: involvement of A2 purinergic receptor subtypes. Life Sci
1999;64:1661-74

13. Belletti S, Uggeri J, Gatti R, Govoni P, Guizzardi S. Polydeoxyribonucleotide promotes cyclobutane pyrimidine dimer repair in UVB-exposed dermal fibroblasts. Photodermatol Photoimmunol Photomed 2007;23:242-9.

14. Khoa ND, Montesinos MC, Reiss AB, Delano D, Awadallah N, Cronstein $\mathrm{BN}$. Inflammatory cytokines regulate function and expression of adenosine A(2A) receptors in human monocytic THP-1 cells. J Immunol 2001;167:4026-32.

15. Chan ES, Fernandez P, Merchant AA, Montesinos MC, Trzaska S, Desai A, et al. Adenosine A2A receptors in diffuse dermal fibrosis: pathogenic role in human dermal fibroblasts and in a murine model of scleroderma. Arthritis Rheum 2006;54:2632-42.

16. Nguyen DK, Montesinos MC, Williams AJ, Kelly M, Cronstein BN. Thl cytokines regulate adenosine receptors and their downstream signaling elements in human microvascular endothelial cells. J Immunol 2003;171:3991-8.

17. Yegutkin GG. Nucleotide- and nucleoside-converting ectoenzymes: important modulators of purinergic signalling cascade. Biochim Biophys Acta 2008;1783:673-94.

18. Montesinos MC, Desai A, Chen JF, Yee H, Schwarzschild MA, Fink JS, et al. Adenosine promotes wound healing and mediates angiogenesis in response to tissue injury via occupancy of $\mathrm{A}(2 \mathrm{~A})$ receptors. Am J Pathol 2002;160:2009-18.

19. Victor-Vega C, Desai A, Montesinos MC, Cronstein BN. Adenosine $\mathrm{A} 2 \mathrm{~A}$ receptor agonists promote more rapid wound healing than recombinant human platelet-derived growth factor (Becaplermin gel). Inflammation 2002;26:19-24.

20. Kim JY, Pak CS, Park JH, Jeong JH, Heo CY. Effects of polydeoxyribonucleotide in the treatment of pressure ulcers. J Korean Med Sci 2014;29 Suppl 3:S222-7.

21. Polito F, Bitto A, Galeano M, Irrera N, Marini H, Calo M, et al. Polydeoxyribonucleotide restores blood flow in an experimental model of ischemic skin flaps. J Vasc Surg 2012;55:479-88.

22. De Aloe G, Rubegni P, Biagioli M, Taddeucci P, Fimiani M. Skin graft donor site and use of polydeoxyribonucleotide as a treatment for skin regeneration: a randomized, controlled, double-blind, clinical trial. Wounds 2004;16:258-63.

23. Gallant-Behm CL, Mustoe TA. Occlusion regulates epidermal cytokine production and inhibits scar formation. Wound Repair Regen 2010;18:235-44.

24. Ravenscroft MJ, Harker J, Buch KA. A prospective, randomised, controlled trial comparing wound dressings used in hip and knee surgery: Aquacel and Tegaderm versus Cutiplast. Ann R Coll Surg Engl 2006;88:18-22. 